\section{Tendência do aleitamento materno no Brasil no último quarto do Século XX}

\section{Trends of breastfeeding in Brazil in the last quarter of the $20^{\text {th }}$ century}

\section{Maria Cristina Ferreira Sena' \\ Eduardo Freitas da Silva ${ }^{2}$ Maurício Gomes Pereira ${ }^{3}$}

'Escola Superior de Ciências da Saúde, Medicina, Fundação de Ensino e Pesquisa em Ciências da Saúde. Brasília, DF

${ }^{2}$ Departamento de Estatística, Instituto de Ciências Exatas, Universidade de Brasília

${ }^{3}$ Faculdade de Ciências da Saúde, Programa de Pós-Graduação em Ciências da Saúde, Universidade de Brasília

Correspondência: Maria Cristina Ferreira Sena, SHIN QI 07 conjunto 3 casa 12 - Lago Norte, Brasília, DF CEP 71515-030

E-mail:mcfsena@yahoo.com

\section{Resumo}

Objetivo: analisar a evolução do aleitamento materno no Brasil de 1974-1975 a 1999. Método: foram comparados três inquéritos populacionais realizados em 1974-1975, 1989, 1999. Os três estudos variaram quanto aos objetivos, plano amostral e coleta de dados, mas foi constante a definição de aleitamento materno. Os resultados das duas primeiras pesquisas foram retirados de trabalho que analisou a evolução do aleitamento materno no período correspondente. Os do terceiro foram gerados por nova análise do banco de dados do estudo original. A fim de permitir a comparação direta dos resultados desta análise com os demais, utilizou-se o modelo probito. A evolução do aleitamento materno nos três pontos no tempo aqui considerados foi verificada pelo método gráfico e por números-índice. Resultados: o aumento da freqüência da amamentação, no período de 25 anos decorridos entre a primeira e a terceira pesquisa, correspondeu a $40 \%$ no grupo de crianças com um mês de vida, a $150 \%$, no quarto mês, a $200 \%$, no sexto mês, e a $240 \%$ aos 12 meses. Conclusão: persistiu nos anos de 1990 a tendência de aumento da prática da amamentação verificada no país no período de 1974-1975 a 1989.

Palavras-chave: Aleitamento materno. Tendência. Brasil 
Abstract

Objective: to analyze the development of Brazilian breastfeeding from 1974-1975 to 1999. Method: three population-based surveys carried out in 1974-1975, 1989, and 1999 were compared. Although they had different objectives, sampling plans, and data collection methods, the definition of breastfeeding was the same. The results of the first two surveys were taken from the original articles. For the 1999 survey, a new analysis was performed in order to allow direct comparison of results. The probit model was used to estimate prevalence in all three studies. The development of breastfeeding was examined through charts and index numbers. Results: the increase in the frequency of breastfeeding, in the period of 25 years between the first and third studies, started at $40 \%$ in the group of children at one month of age, to $150 \%$ at the fourth month of age, to $200 \%$ at the sixth month of age, and to $240 \%$ at the $12^{\text {th }}$ month of age. Conclusion: the increasing trend in the prevalence of breastfeeding verified in Brazil in 19741975 to 1989 continued in the 1990's.

Keywords: Breastfeeding. Trends. Brazil

\section{Introdução}

O declínio do aleitamento materno foi observado em diferentes regiões do mundo nos primeiros três quartos do século $\mathrm{XX}^{1-3}$. Dos países em desenvolvimento, os situados na América Latina apresentaram, na década de 1970, as menores durações de aleitamento materno ${ }^{3}$. No Brasil, o aumento da desnutrição e da mortalidade infantil, verificados nessa época em várias localidades, alertou as autoridades sanitárias para o uso de leite não-humano na alimentação de menores de um $\mathrm{ano}^{4}$. Tal fato motivou a realização de ações formais e sistemáticas, a partir de 1981, para fortalecer a prática do aleitamento materno no país ${ }^{5,6}$.

Como parte desse processo, sentiu-se a necessidade de acompanhar a tendência da população em relação à alimentação recomendada para o primeiro ano de vida $^{7}$.

Comparação dos resultados de duas pesquisas brasileiras, realizadas em meados da década 70 e no fim dos anos $1980^{8}$, mostrou aumento da duração mediana do aleitamento materno, de 75 dias para 167 dias no período analisado. Uma outra investigação de âmbito nacional foi promovida em 1999 para avaliar os indicadores de amamentação na área urbana do Brasil ${ }^{9}$. O presente trabalho faz nova análise dos dados coletados em 1999 e os compara com as taxas de 1974-1975 e de 1989, de modo a descrever a tendência do aleitamento materno no período.

\section{Método}

As pesquisas utilizadas neste trabalho têm como aspectos metodológicos comuns serem estudos transversais, de base populacional, realizados em amostras probabilísticas e de abrangência nacional ${ }^{8-}$ ${ }^{11}$. As características das investigações estão resumidas na Tabela 1. Embora elas não tivessem os mesmos objetivos, foi possível obter dados semelhantes para aleitamento materno. 
Tabela 1 - Características das pesquisas comparadas

Table 1- Main topics of the surveys studied

\begin{tabular}{|c|c|c|c|c|c|c|c|}
\hline $\begin{array}{l}\text { Ano da } \\
\text { pesquisa }\end{array}$ & $\begin{array}{l}\text { Local e data } \\
\text { da coleta } \\
\text { de dados }\end{array}$ & $\begin{array}{l}\text { Objetivo } \\
\text { da pesquisa } \\
\text { original }\end{array}$ & $\begin{array}{l}\text { Etapas da } \\
\text { composição } \\
\text { da amostra } \\
\text { na pesquisa } \\
\text { original }\end{array}$ & $\begin{array}{l}\text { Amostra } \\
\text { de crianças } \\
\text { menores de } \\
\text { um ano }\end{array}$ & $\begin{array}{l}\text { Obtenção } \\
\text { do dado de } \\
\text { aleitamento } \\
\text { materno }\end{array}$ & $\begin{array}{l}\text { Análise } \\
\text { estatística }\end{array}$ & $\begin{array}{l}\text { Programa } \\
\text { estatístico }\end{array}$ \\
\hline $1999^{9,12}$ & $\begin{array}{l}\text { Postos de } \\
\text { vacinação } \\
16 \text { de outubro }\end{array}$ & $\begin{array}{l}\text { Situação do } \\
\text { aleitamento } \\
\text { materno na } \\
\text { área urbana } \\
\text { das capitais } \\
\text { de estado e } \\
\text { no Distrito } \\
\text { Federal }\end{array}$ & $\begin{array}{l}\text { 1. Seleção } \\
\text { aleatória } \\
\text { dos postos } \\
\text { de vacinação } \\
\text { 2. Seleção } \\
\text { sistemática } \\
\text { de crianças } \\
\text { na fila }\end{array}$ & 48.845 & $\begin{array}{l}\text { Questionário. } \\
\text { Às mães foi } \\
\text { perguntado se } \\
\text { nas últimas } \\
24 \text { horas a } \\
\text { criança fora } \\
\text { alimentada } \\
\text { com leite } \\
\text { materno, água, } \\
\text { chá, sucos, outro } \\
\text { leite, frutas, } \\
\text { sopas e refeição } \\
\text { da família. }\end{array}$ & $\begin{array}{l}\text { Regressão } \\
\text { probito }\end{array}$ & $\begin{array}{l}\text { SAS } \\
\text { Versão } 8.2\end{array}$ \\
\hline $1989^{8,11}$ & $\begin{array}{l}\text { Domicílios } \\
\text { julho a } \\
\text { setembro }\end{array}$ & $\begin{array}{l}\text { Estado } \\
\text { nutricional } \\
\text { da população } \\
\text { brasileira }\end{array}$ & $\begin{array}{l}\text { 1. Seleção } \\
\text { aleatória dos } \\
\text { setores } \\
\text { censitários } \\
\text { 2. Seleção } \\
\text { aleatória dos } \\
\text { domicílios }\end{array}$ & $1.431^{*}$ & Questionário & $\begin{array}{l}\text { Regressão } \\
\text { probito }\end{array}$ & $\begin{array}{l}\text { Próprio do } \\
\text { grupo de } \\
\text { pesquisa que } \\
\text { propôs o } \\
\text { modelo probito } \\
\text { para estimar } \\
\text { indicadores de } \\
\text { aleitamento } \\
\text { materno }\end{array}$ \\
\hline $1974-1975^{8,10}$ & $\begin{array}{l}\text { Domicílios } \\
\text { agosto-agosto }\end{array}$ & $\begin{array}{l}\text { Consumo } \\
\text { alimentar } \\
\text { das famílias } \\
\text { brasileiras }\end{array}$ & $\begin{array}{l}\text { 1. Seleção } \\
\text { aleatória dos } \\
\text { setores } \\
\text { censitários } \\
\text { 2. Seleção } \\
\text { aleatória dos } \\
\text { domicílio }\end{array}$ & $7.591^{*}$ & $\begin{array}{l}\text { Questionário } \\
\text { Às mulheres } \\
\text { foi perguntado } \\
\text { se estavam } \\
\text { grávidas ou } \\
\text { amamentando. } \\
\text { O dado permitiu } \\
\text { analisar os } \\
\text { menores de } \\
\text { um ano em } \\
\text { aleitamento } \\
\text { materno }\end{array}$ & $\begin{array}{l}\text { Regressão } \\
\text { probito }\end{array}$ & $\begin{array}{l}\text { Próprio do } \\
\text { grupo de } \\
\text { pesquisa que } \\
\text { propôs o } \\
\text { modelo probito } \\
\text { para estimar } \\
\text { indicadores de } \\
\text { aleitamento } \\
\text { materno }\end{array}$ \\
\hline
\end{tabular}

* Essas pesquisas incluíram adultos e crianças. Na coluna só está assinalado o número de menores de um ano, que é a população de interesse para avaliar o aleitamento materno.

* The studies included adults and children. In the column only the number of individuals less than one year of age is pointed out, that is, the population of interest to assess breastfeeding.

O aleitamento materno refere-se à condição de a criança ser alimentada com leite materno por ocasião dos inquéritos analisados (current breastfeeding status) ${ }^{2}$, independentemente de receber outros alimentos $^{8,9}$.

As informações dos dois primeiros inquéritos foram obtidas no trabalho de Venâncio e Monteiro ${ }^{8}$. As do terceiro foram geradas por meio de nova análise do banco 
de dados do estudo original, realizada pelos autores do presente artigo, de modo a tornar comparáveis os resultados nos três pontos no tempo aqui considerados.

As freqüências de aleitamento materno foram estimadas por ponto e por intervalo (IC 95\%) nas idades selecionadas de 1, 4, 6 e 12 meses, utilizando-se o modelo probito $^{8,12-14}$.

A tendência das taxas de aleitamento materno foi analisada pelo método gráfico e por meio de números-índice ${ }^{15}$. O período de 1974-1975 foi definido como o ano base para comparação e a ele atribuído o número-índice "100”. Para os anos de 1989 e 1999, os cálculos foram feitos dividindose as prevalências de aleitamento materno dos respectivos anos pelas prevalências do ano base. Os valores obtidos expressam as variações das taxas de aleitamento materno nas décadas de 1980 e de 1990 em relação às da década de 1970.

\section{Resultados}

A Figura 1 apresenta graficamente a sín- tese dos resultados encontrados. Cada linha representa uma pesquisa. Observa-se que houve aumento considerável do percentual de crianças em aleitamento materno nas idades estudadas. As prevalências são muito mais altas na última pesquisa, em qualquer das idades, talvez com exceção do primeiro mês de vida em que as duas investigações recentes mostram valores próximos. Vê-se ainda que houve diminuição dos declives dos segmentos de reta relativos às idades de 1 a 4 meses e de 4 a 6 meses.

Os números utilizados para compor a Figura 1 estão na Tabela 2, acompanhados da precisão da estimativa. Nota-se que os intervalos de confiança são bem mais estreitos na pesquisa de 1999, visto estarem baseados em grande tamanho de amostra.

A Tabela 3 apresenta os números-índices. Os valores indicam que o aumento da freqüência da amamentação, no período total de 25 anos decorridos entre a primeira e a terceira pesquisa, correspondeu a $40 \%$ no grupo de crianças com um mês de vida, a $150 \%$, no quarto mês, a $200 \%$, no sexto mês, e a $240 \%$ aos 12 meses.

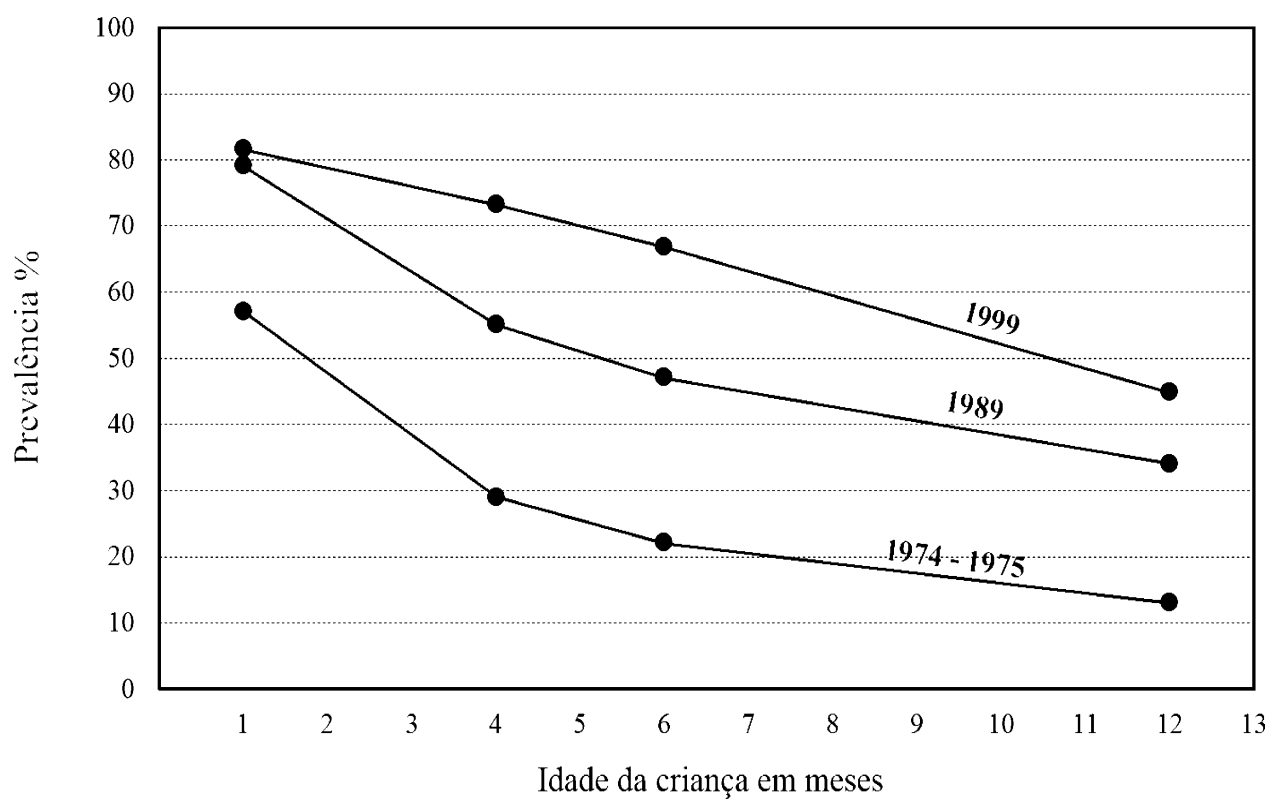

Figura 1 - Prevalência do aleitamento materno nas idades de 1, 4, 6 e 12 meses, Brasil urbano 1974-1975, 1989 e 1999

Figure 1 - Prevalence of breastfeeding in Brazilian urban areas in the years of 1974-1975, 1989 and 1999 for children at the ages of 1, 4, 6, and 12 months 
Tabela 2 - Prevalência de aleitamento materno, no Brasil urbano em 1974 - 1975, 1989 e 1999, expressa em percentual e intervalo de confiança de $95 \%$

Table 2- Prevalence of breastfeeding in Brazilian urban areas in the years of 1974-1975, 1989, and 1999, by percentage and $95 \%$ confidence interval

\begin{tabular}{|c|c|c|c|c|c|c|c|c|}
\hline \multirow{3}{*}{$\begin{array}{l}\text { Ano da } \\
\text { pesquisa }\end{array}$} & \multicolumn{8}{|c|}{ Idade da criança em meses } \\
\hline & \multicolumn{2}{|r|}{1} & \multicolumn{2}{|r|}{4} & \multicolumn{2}{|r|}{6} & \multicolumn{2}{|c|}{12} \\
\hline & $\%$ & (IC 95\%) & $\%$ & (IC 95\%) & $\%$ & (IC 95\%) & $\%$ & (IC 95\%) \\
\hline 1999 & 81,5 & $(80,9-82,1)$ & 73,3 & $(72,8-73,7)$ & 66,8 & $(66,4-67,7)$ & 44,8 & $(43,9-45,8)$ \\
\hline $1989^{*}$ & 79 & $(66-89)$ & 55 & $(48-63)$ & 47 & $(40-55)$ & 34 & $(25-45)$ \\
\hline $1974-1975^{*}$ & 57 & $(53-62)$ & 29 & $(27-31)$ & 22 & $(20-24)$ & 13 & $(11-15)$ \\
\hline
\end{tabular}

* Fonte: Venâncio e Monteiro, 1998/* Source: Venâncio e Monteiro, 1998

Tabela 3 - Prevalência de aleitamento materno, no Brasil urbano em 1974-1975, 1989 e 1999, expressa em percentual e em números-índice

Table 3 - Prevalence of breastfeeding in Brazilian urban areas in the years of 1974-1975, 1989 and 1999 years, by percentage and index numbers

\begin{tabular}{|c|c|c|c|c|c|c|c|c|}
\hline \multirow{3}{*}{$\begin{array}{l}\text { Ano da } \\
\text { pesquisa }\end{array}$} & \multicolumn{8}{|c|}{ Idade da criança em meses } \\
\hline & \multicolumn{2}{|c|}{1} & \multicolumn{2}{|r|}{4} & \multicolumn{2}{|c|}{6} & \multicolumn{2}{|c|}{12} \\
\hline & $\%$ & $\begin{array}{l}\text { Números- } \\
\text { índice }\end{array}$ & $\%$ & $\begin{array}{l}\text { Números- } \\
\text { índice }\end{array}$ & $\%$ & $\begin{array}{l}\text { Números- } \\
\text { índice }\end{array}$ & $\%$ & $\begin{array}{l}\text { Números- } \\
\text { índice }\end{array}$ \\
\hline 1999 & 81,5 & 140 & 73,3 & 250 & 66,8 & 300 & 44,8 & 340 \\
\hline $1989^{*}$ & 79 & 140 & 55 & 190 & 47 & 210 & 34 & 260 \\
\hline $1974-1975^{*}$ & 57 & 100 & 29 & 100 & 22 & 100 & 13 & 100 \\
\hline
\end{tabular}

* Fonte: Venâncio e Monteiro, 1998/* Source: Venâncio e Monteiro, 1998

\section{Discussão}

Três pesquisas nacionais realizadas no fim do século XX, compreendendo um período de 25 anos, mostram aumento da prática da amamentação nas crianças menores de um ano de idade no país. As prevalências em 1999 foram maiores do que as verificadas em 1989, e essas superiores às da década de 1970.

As amostras estudadas são probabilísticas, de abrangência nacional, mas diferem no seu tamanho. As investigações das décadas de 70 e de 80 são estudos amplos envolvendo crianças e adultos. Quando são enfocadas somente as crianças nessas pesquisas, os respectivos tamanhos de amostra (7.591 e 1.431 crianças) são bem inferiores aos do inquérito de 1999 (48.845 crianças). Embora os efetivos sejam grandes, em todas as pesquisas, a estratificação da amostra para cada mês de idade no primeiro ano de vida, faz com que os intervalos de confiança sejam amplos em 1989, médios em 1974-1975 e pequenos em 1999 (Tabela 2).

Dois aspectos positivos foram o uso das mesmas idades para comparação das taxas e da mesma modelagem estatística para análise da tendência aqui apresentada. O emprego de modelos estatísticos distintos para o estudo de um mesmo problema pode gerar estimativas diferentes. Esse fato não implica erros, mas limita a comparação direta dos resultados.

A definição de aleitamento materno foi semelhante nos três estudos. No entanto, houve variação no modo de coletar os dados. Os questionários utilizados foram específicos de cada pesquisa. Nas duas primeiras investigações, as entrevistas foram realizadas em domicílio, sendo que a pri- 
meira estendeu-se por um ano e a segunda por três meses. Na última, os dados foram obtidos em um único dia durante a Campanha Nacional de Vacinação, cuja cobertura aproxima-se de $100 \%$ em todo o país.

A situação de aumento da freqüência da amamentação no Brasil também foi verificada nas pesquisas de demografia e saúde, as $D H S$, realizadas no período de 1986 a 1999, no Brasil e em outros países da América Latina e Caribe ${ }^{16}$. De seis países com mais de duas $D H S$ realizadas, apenas na Guatemala houve pequena redução na duração mediana da amamentação. Nos demais - Brasil, Bolívia, Colômbia, Peru e República Dominicana - houve aumento. O acréscimo ocorreu nos meios urbano e rural, sendo mais intenso na área urbana.

Análises epidemiológicas de tendênci- as, como a aqui apresentada, são valiosas, pois produzem subsídios para guiar o planejamento de ações e serviços para a população. Políticas de proteção e programas de promoção do aleitamento materno podem ser possíveis fatores explicativos para a melhora verificada na prevalência de aleitamento materno com o passar do tempo. A melhora da situação sugere o deslocamento do desmame para idades maiores, o que é desejável. Aponta também para a extensão do aleitamento materno para além do primeiro ano de vida, o que também é desejável.

Em conclusão, a análise acrescenta em uma década o conhecimento da tendência de aleitamento no país. Embora o aumento da prevalência do aleitamento materno no país seja progressivo e persistente, ainda há espaço para melhoras.

\section{Referências}

1. Hendershot GE. Trends in breast-feeding. Pediatrics 1984; 74(4S): 591-602.

2. Notzon F. Trends in infant feeding in developing countries. Pediatrics 1984; 74(4S): 648-66.

3. Victora C, Albernaz E, Lutter C. The role of research in the formulation of infant feeding policies in Latin America. In: Freire WB, editor. Nutrition and active life: from knowledge to action. Washington, DC: Pan American Health Organization; 2005. p. 21-9 (Scientific and Technical Publication, 612).

4. Ministério da Saúde (Br). Programa nacional de incentivo ao aleitamento materno. Brasília, DF: INAN; 1991.

5. Marin P, Oliveira YP. The breastfeeding programme in Brazil. In: Programmes to promote breastfeeding. New York. Oxford University Press 1988: 153-60.

6. Rea MF, Araújo MFM. Implementation of breast-feeding practices in Brazil: from international recommendations to local policy. In: Freire WB, editor. Nutrition and active life: from knowledge to action. Washington, DC: Pan American Health Organization; 2005. p. 109-30 (Scientific and Technical Publication, 612).

7. Programmes proposed by UNICEF in support of breastfeeding. In: Assignment children: a journal concerned with children, women and youth in development. $2^{\text {a }}$ ed. Geneve: UNICEF; 1981; 55/56: 19-24 (Dossier).
8. Venâncio SI, Monteiro CA. A tendência da prática da amamentação no Brasil nas décadas de 70 e 80. Rev Bras Epidem 1998; 1(1): 40-9.

9. Ministério da Saúde. Secretaria de Políticas de Saúde. Prevalência do aleitamento materno nas capitais brasileiras e no Distrito Federal. Brasília; 2001.

10. Viacava F, Figueiredo CMF, Oliveira WA. A desnutrição no Brasil uma análise do Estudo Nacional de Despesa Familiar (IBGE 74/75) para o nordeste, Estado de São Paulo e Estado do Rio de Janeiro. Petrópolis: Editora Vozes, Co-Editora FINEP; 1983. p. 161-9.

11. Leão MM, Coitinho DC, Recine E, Costa LAL, Lacerda AJ. O Perfil do aleitamento materno no Brasil. In: Monteiro MFG, Cervini R, (Org.). Perfil estatístico de crianças e mães no Brasil: aspectos de saúde e nutrição de crianças no Brasil 1989. Rio de Janeiro: IBGE/Departamento de Estatísticas e Indicadores Sociais; 1992. p. 97-109.

12. SAS Institute Inc., SAS/STAT ${ }^{\circledR}$ Software: Changes and Enhancements, release 8.2. Cary, NC: SAS Institute Inc., 2001.

13. Ferreira UM, Cardoso MA, Ferreira CS, Szarfare SC. Rapid epidemiologic assessment of breastfeeding practices: probit analysis of current status data. J Trop Pediatr 1996; 42: 50-3. 
14. Sena MCF. Prevalência do aleitamento materno exclusivo no Distrito Federal e sua associação com o trabalho materno fora do lar [dissertação de mestrado]. Brasília: Universidade de Brasília; 1997.

15. Pereira MG. Variáveis relativas ao tempo. In: Pereira MG. Epidemiologia: teoria e prática. $1^{\text {a }}$ ed. Rio de Janeiro: Guanabara Koogan; 2000. p. 245-66.
16. Pérez-Escamilla, R. Breastfeeding and the nutritional transition in the Latin American and Caribbean Region: a success story? Cad Saúde Pública 2003; 19 (S1): 119-27.

Recebido em: 15/01/07 Versão final reapresentada em: 14/08/07 Aprovado em: 03/10/07 